\section{Mentalità stratigrafica e progetti per la conoscenza e per la conservazione}

\author{
Gianfranco Pertot*, Gian Paolo Treccani** \\ * Politecnico di Milano - Facoltà di Architettura \\ * Università di Brescia - Facoltà di Ingegneria
}

\begin{abstract}
Riassunto
Gli autori presentano i risultati più significativi di una ricerca svolta presso le Università di Milano e di Brescia, nell'ambito dei corsi di Restauro architettonico. Fra i principali obiettivi dello studio e delle esperienze didattiche correlate vi è il tentativo non solo di utilizzare le procedure dell'archeologia stratigrafica, per quanto utile e possibile, nella complessa serie di operazioni che segna la redazione di un progetto di conservazione e di riuso, ma di trasferirvi anche quella che si può definire come "mentalità stratigrafica". In particolare considerando nuovi codici, quali le Interfacce di Fase e le Unità Stratigrafiche Associate. A corredo di queste argomentazioni vengono anche svolte alcune considerazioni in merito al cosiddetto "Restauro archeologico".
\end{abstract}

Parole chiave: Restauro Architettonico, Mentalità stratigrafica.

\section{Abstract}

The present work is made to show a didactic experience acquired by the authors at the Universities of Milan and Brescia (Italy) in the sphere of teaching Architectural Restoration. The peculiar aspect of research is the attempt to apply not only the procedures of archeological stratigraphy but also the "stratigraphical mentality" to the project of conservation and re-use of architecture. Introducing and using, for example, new models of archeological stratigraphy lecture of the buildings expressed for the requirements of conservation and re-use, in proposing the new index-codes of Phases Intersides and Associated Stratigraphical Unity. The authors finally put forward some comments about the so called "archaological" Restoration.
\end{abstract}

Key words: Architectural Restoration, Stratigraphical mentality.
Il contributo che si presenta in questa sede propone un bilancio di un'attività di ricerca che ha avuto inizio nel 1994 presso la Facoltà di architettura del Politecnico di Milano ed è stata ulteriormente incrementata negli anni seguenti, con primi contributi a partire dal biennio 199596, momento di nascita della rivista Archeologia dell'architettura e segnato dallo svolgimento, in Italia, di convegni e seminari sui rapporti fra archeologia ed architettura ${ }^{1}$.

Studi e sperimentazioni didattiche, condotte in seno a corsi di Laboratorio di Restauro Architettonico e in occasione della redazione di tesi di laurea in Architettura, sono state mirate principalmente ad approfondire aspetti specifici insiti sia nell'applicazione delle tecniche di indagine archeologica agli edifici esistenti, sia nell'articolazione del rapporto fra il rilevamento stratigrafico ed il progetto di conservazione e di riuso, con risultati che sono confluiti in una recente pubblicazione ${ }^{2}$.

Sono state in primo luogo raccolte le indicazioni provenienti dalle prime applicazioni dell'analisi stratigrafica ai manufatti edilizi, intesi come bacino di sedimentazione tridimensionale, in diverse occasioni.

I fronti di ricerca aperti sono stati poi indirizzati verso la verifica della possibilità, liceità ed utilità dell'applicazione dello strumento stratigrafico nell'ambito della disciplina dello studio e del restauro degli edifici esistenti. Ma contemporaneamente si è affrontato con curiosità un altro aspetto, affrontando un approfondimento teoretico inerente radici comuni, differenze, elementi caratterizzanti dell'opera e del pensiero degli archeologi e degli architetti nei momenti in cui le due discipline e le loro articolazioni si sono effettivamente confrontate con le problematiche teoriche e pratiche dello studio e della conservazione dei manufatti esistenti.

\section{RESTAURO “ARCHEOLOGICO”?}

La prossimità e la condivisione di alcuni obiettivi fra archeologia e restauro vantano in Italia (e non solo) un'origine lontana. Da un lato è inevitabile riandare ai primi esercizi dei restauratori per la ricomposizione dell'antichità magno greca (tempio di Segesta) e soprattutto dell'antichità romana classica (gli esempi paradigmatici sempre ricordati sono gli interventi dei primi anni

\footnotetext{
${ }^{1}$ Patrimonio archeologico e progetto architettonico e urbano (Milano, 21-22 maggio 1996), Dal sito archeologico all'archeologia del costruito. Conoscenza, progetto e conservazione (Bressanone, 3-6 luglio 1996), Archeologia e restauro dei monumenti: dieci anni dopo (Certosa di Pontignano, 21-22 marzo 1997), giornate di studio dedicate al tema in Brescia (2 aprile 1996), Genova (9 maggio 1996), Bordighera (30 settembre 1996), Monte Barro (12-13 luglio 1997).

2 TrecCani 2000, pubblicazione realizzata con il contributo del Ministero per l'Università e la Ricerca Scientifica e Tecnologica (MURST).
} 
dell'Ottocento a Roma, sul Colosseo e sull'Arco di Tito). Dall'altro è innegabile che alcuni aspetti della mentalità archeologica (per esempio l'attenzione al dato materiale, o anche alcune tecniche di analisi e di rappresentazione) sembrano essere elementi di convergenza sui quali si sono orientati precocemente già alcuni architetti ottocenteschi e ai quali il restauro è spesso riandato, pur con esiti diversi. Né si può dimenticare di come nei primi anni dell'Unità nazionale italiana gli organismi preposti alla tutela si occupassero indistintamente di archeologia e di architettura fino alle più nette distinzioni introdotte nell'ultimo decennio del XIX secolo, termine che segna forse anche il venire meno di una figura di conoscitore tanto di architettura come di archeologia e di storia, incarnata da molte importanti figure (Alfredo D'Andrade, Luca Beltrami, Giacomo Boni, Camillo Boito, per restare nell'ambito dei più noti), e più tardi ancora evocata negli scritti di Gustavo Giovannoni.

Per contro l'archeologia porta innegabilmente con sé, dall'origine, una vocazione alla rimozione del deposito coniugata con la tendenza a fissare i limiti di questa rimozione sulla base di criteri cronologico-artistici.

Siamo tutti a conoscenza di come tanti importanti cantieri archeologici siano stati intenzionalmente e pervicacemente condotti (e lì conclusi, a meno di piccoli ulteriori saggi stratigrafici), alla fase o agli strati considerati rappresentativi del momento di maggior fulgore o di maggior estensione della collettività che un tempo abitò il sito. Il debito di questo atteggiamento nei confronti dello storicismo e della storia dell'arte (classica in particolare) è palese. Per contro, dal momento in cui l'analisi archeologica ha assunto una veste più rigorosa (soprattutto, ma non solo, in tempi recenti) è stata prontamente assunta (in particolare per lo studio degli elevati) come operatore "oggettivo". Non di rado questa assunzione ha ispirato interventi ostensivi, dimostrativi di una conoscenza data per raggiunta in veste definitiva ed esibita sotto forma di ricostituiti palinsesti. Operazione che contribuisce ad interrompere la sequenza vitale del manufatto, azzerandola e dandone al contempo un'interpretazione che si austostima come "oggettiva", grazie proprio al rigore metodologico impiegato (solo) nella fase di analisi e alla veste, per così dire, anatomica della presentazione. Questo tipo di intervento parla evidentemente più del metodo impiegato, esplicitandone i risultati, che del manufatto.

L'anello debole di questa catena operativa (i cui esempi si sprecano, tanto nel campo dell'intervento sui resti archeologici quanto in quello dell'intervento sugli edifici, soprattutto sulle facciate) è evidentemente rappresentato da una negazione della potenziale multiformità delle possibilità conoscitive e dei percorsi e dei tempi della conoscenza. Un corollario a queste posizioni è la fortunata tradizione della selezione intesa come espunzione delle parti ritenute non pertinenti al corpus originario di un manufatto o estranee alle facies ritenute più interessanti o di maggior valore storico e/o artistico. Tradizione viva nelle due discipline, e contro la quale l'analisi stratigrafica può essere contraddittoriamente assunta come un baluardo oppure come un ulteriore strumento vivisettivo e giustificativo di scelte operative selettive, certificando il valore superfetativo delle aggiunte.

Posto invece che "la potenzialità testimoniale è indeterminabile" e che è "altrettanto indeterminabile il numero dei percorsi di ricostruzione storiografica che è possibile definire, o quello delle modalità di conoscenza, i cui orizzonti sono in continuo ampliamento" (BeLlinI, 1996: 2) riteniamo sia inaccettabile e pericoloso, oltre che velleitario, ogni tentativo di oggettivazione di uno sguardo unilaterale sulle testimonianze del passato.

Coerentemente con questi assunti, la proposizione di un unico percorso storiografico, sia pure fondato sulla cronologia relativa indicata dalla lettura stratigrafica, risulta comunque arbitraria.

Nonostante queste derive, il restauro in ambito archeologico viene considerato generalmente più attento rispetto alla materialità del manufatto. In molti casi effettivamente questa cautela, diversamente motivata, si è riscontrata, mentre in molti altri è stata pesantemente disattesa, non solo nel campo della statuaria classica, che vanta esempi paradigmatici, ma anche in quello architettonico, soprattutto grazie alle tante parziali anastilosi che segnano invariabilmente il passaggio dal termine delle campagne di scavo al momento della presentazione del sito archeologico.

Alla luce di queste e di altre considerazioni sugli intrecci fra restauro e archeologia e, nella fattispecie, sul restauro in ambito archeologico, è stata avviata una verifica sottoponendo le pagine di Notizie degli Scavi di Antichità (la rivista italiana che dal 1876 pubblica i resoconti di scavi e di ritrovamenti archeologici e gli studi sui materiali rinvenuti) alla ricerca di indicatori semantici e di metodo, oltre che di veri e propri resoconti su campagne di restauro intraprese da archeologi o da architetti nel cantiere di scavo ${ }^{3}$.

I risultati sono stati articolati intorno a tematiche che le due discipline condividono o dalle quali vengono o

\footnotetext{
${ }^{3}$ Un primo tentativo di sistematizzare i dati raccolti è in PERTOT, 2000.
} 
sono state attraversate. Ne è scaturito un panorama più che variegato, che ha avuto il merito, se così si può dire, di disporre atti e intenzioni su un terreno comune e di consentire un confronto critico ${ }^{4}$.

Questo terreno talvolta è stato solo semanticamente tale, costituendosi attorno a parole chiave (si pensi ai tanti diversi esiti della declinazione della pratica dell'anastilosi), ad espressioni e a indicatori disciplinari e culturali, rapportati agli interventi e alla cultura dei singoli attori e del loro tempo.

Talvolta invece è stato individuato in siti (Pompei, Ostia, i fori di Roma) sui quali si sono succeduti gli operatori, e con essi filosofie e tecniche di intervento simili o diverse. Altre volte ancora sono stati considerati gli strumenti del lavoro dell'archeologo e del restauratore, quelli dello scavo ma anche quelli della rappresentazione (rilievi, disegni, ...) e delle indagini diagnostiche.

Oppure è stato cercato nella presenza di protagonisti implicati, in senso positivo, su diversi fronti, rinvenendo anche contributi, seppur limitati, decisamente poco noti (Beltrami, Pane ...), concorrendo, si spera (sia pure con un taglio particolare e con un contributo quantitativamente minimo), anche alla problematica costruzione di un apparato storiografico sull'archeologia italiana moderna.

In estrema sintesi, alla fine di questo percorso di analisi e di ricerca, si ravvisa l'inopportunità di definire un "restauro archeologico" come area autonoma, come spesso è stato fatto, intendendo una pratica di intervento sull'antico caratterizzata da criteri propri e peculiari, anche ritenuti più "oggettivi” o maggiormente specialistici (o specializzati) rispetto a quelli impiegati in campo architettonico.

Meglio sottolineare, invece, come il rapporto fra lo studio dell'antico (dell'esistente) e le pratiche del suo restauro non è e non può essere monodirezionale, bensì si organizza intorno a tanti modi distinti ed altrettanti sguardi soggettivi. Ed è interessante interrogarsi, oggi, sull'orizzonte di senso in cui si situa un loro plausibile incontro.

\section{MENTALITÀ STRATIGRAFICA}

Ritornando al tema dell'impiego di tecniche archeologiche nel campo degli interventi sul costruito, di fronte al diffuso interesse per il carattere di novità apportato dalla diffusione dell'analisi stratigrafica non ci si può esimere dal considerare il pericolo insito in una trasposizione meccanica della logica stratigrafica nella progettazione. L'impiego

\footnotetext{
${ }^{4}$ Una ricerca analoga ha avuto invece come riferimento i contributi dedicati a restauri di manufatti archeologici apparsi sulle riviste di architettura a partire dagli anni Venti del XX secolo (OTERI, 2000)
}

stesso della stratigrafia nel lungo ed articolato processo di progettazione di interventi sul costruito esistente richiede una serie non indifferente di correttivi.

Indubbiamente gli aspetti positivi sono parecchi, a cominciare dall'apparente efficacia ed efficienza del metodo, in grado di consegnare all'architetto un quadro in cui, teoricamente, ogni parte della fabbrica acquisisce una distinta e riconosciuta individualità; il che ne spiega la fortuna presso coloro, architetti conservatori in primo luogo, che sono stati e sono alla ricerca di strumenti analitici sempre più raffinati in grado di dar conto della complessità dei manufatti, di uscire da schematismi e da generalizzazioni di tipo selettivo e aprioristico (tipologismo), di accogliere su carte tematiche riferite ad un'unica base gli apporti di altri studi e di altre discipline e, soprattutto, di mettere a punto interventi il più possibili mirati.

Il rigore logico insito nella sistematica che accompagna l'analisi stratigrafica ha comportato la sua elevazione a tecnica conoscitiva dell'architettura, ritenuta in grado di restituire, a mezzo di un rilievo stratigrafico, un quadro analitico molto più completo, minuzioso, potente, rigoroso rispetto ai rilievi comunemente approntati e, quel che più conta, apparentemente basato su criteri di lettura e rappresentazione oggettivi.

Il rilievo stratigrafico non sfugge però alle indeterminatezze che accompagnano tutti i rilievi, i quali, oltre a patire la difficoltà di dover dar conto di grandezze diverse e complementari (si pensi solo alla compresenza di aspetti qualitativi e di aspetti quantitativi), rivestono, per la stessa natura dell'operazione di rilevamento, un carattere di approssimazione, costituendosi pertanto come opere indirizzate, relative e storicizzabili, impronte digitali della cultura di chi li produce.

A ciò si aggiunga che la lettura stratigrafica si imposta in gran parte sull'esperienza e sulla capacità di osservazione dell'operatore che la effettua.

I risultati sarebbero pertanto da assumere come tutt'altro che commensurabili e sovrapponibili alla varietà informativa dell'esistente.

Ciò non toglie che il fascino per il rigore metodologico dello strumento stratigrafico, pur con le sue difficoltà applicative, ha sancito un inedito quanto fortunato matrimonio di interesse fra archeologia ed architettura che spesso è andato oltre i limiti della reciproca convenienza.

La proposta elaborata in questi anni, e di cui si dà breve cenno, incentrata sull'elaborazione e sulla applicazione di schede di lettura, archiviazione e gestione degli interventi, ha inteso assorbire la logica e la sistematica stratigrafiche all'interno dei loro confini, che rimangono 
prettamente analitici, per trasporre invece la mentalità stratigrafica nel sistema di gestione dei dati di progetto.

Sul piano progettuale il contributo di utilità più evidente consiste certamente in una migliore e più puntuale considerazione delle singole parti della fabbrica, dei materiali costitutivi e delle alterazioni e delle patologie di degrado. Andando a migliorare un consolidato processo conoscitivo e di intervento che per istanza culturale privilegia la materialità dei manufatti, considera la singolarità e la diversità come plusvalore e ne prospetta la permanenza.

Posta questa premessa l'impiego dell'analisi stratigrafica nel quadro dell'intervento sui manufatti esistenti necessita di alcuni integrazioni.

Alcuni sono ampliamenti di tipo tecnico di un orizzonte semantico eccessivamente ristretto (in questo senso si preferisce, per i molteplici orizzonti di senso che sottende, la dicitura Unità stratigrafica costruttiva in luogo della più ambigua dicitura Unità stratigrafica muraria). Oppure procedimenti di sintesi atti a ricondurre nel panorama della progettualità e delle funzioni tecnologiche (Unità stratigrafiche associate) o dei momenti d'uso (Interfacce di fase) gli elementi disaggregati dalla logica decostruttiva dell'analisi stratigrafica.

Potremmo addirittura sancire la distinzione fra i due momenti con un witz dialettico, un po' improprio ma efficace: analisi stratigrafica e sintesi stratigrafica.

Se il ricorso alla logica stratigrafica nelle fasi analitiche può dunque frenare alcune scelte prevaricatrici, non può però automaticamente dettarne di virtuose. Questo è ancora affare della cultura del progettista e della sua capacità di interpretare bisogni, necessità, aspettative, disponibilità e risorse e di confrontare le possibilità di intervento su piani molteplici o, meglio, multicriteriali.

In realtà il rapporto fra archeologia stratigrafica e progetto di conservazione sottende aspetti più sottili e profondi che vanno al di là di una sistematica tecnica e dei procedimenti logici e che autorizzano a dare, se non una dimensione teoretica, almeno un peculiare orizzonte di senso alla definizione di mentalità stratigrafica.

Aspetti che appaiono in consonanza con le riflessioni più mature che si svolgono in seno alla disciplina del restauro architettonico e in sostanza con l'evoluzione metodologica del progetto. Aspetti che sono in consonanza con posizioni già difese da coloro che si occupano di conservazione $e^{5}$ che vale la pena di ricordare brevemente.

In primo luogo l'adozione di procedimenti di analisi stratigrafica, dispositivo in sé analitico e non distruttivo, porta un'apertura decisiva e caratterizzante sul lato materiale/fisico dell'architettura. Un apporto in più, quindi, al riconoscimento della portata e della potenzialità testimoniale insita nell'aspetto materico delle parti dell'architettura indipendentemente dalla loro definizione formale.

Non vi sono poi, nella logica della stratigrafia -come del resto non vi possono essere in quella della conservazione- elementi originali ed elementi non originali, piuttosto che testimonianze di una tecnica costruttiva da considerarsi rilevante oppure no, bensì vi è un intero sistema dove oltre agli oggetti (per lo studio dei quali peraltro non è strettamente necessario un approccio stratigrafico) si considerano le relazioni fra gli oggetti. Ne deriva che ogni forma di discriminazione esercitata con il restauro produce un'erosione irreversibile di questa complessità, minandone la stessa struttura e la possibilità di una sua comprensione. In altri termini dovrebbe essere consequenziale pensare alla stratificazione e alle tracce che la rendono riconoscibile come ad una sorta di plusvalore e non come ad un disvalore da emendare o da correggere.

Così come consequenziale dovrebbe essere l'attribuzione di valore storiografico alla cronologia relativa che riassume il processo di stratificazione (una storia spesso senza date e senza nomi), confermando, se ve ne fosse bisogno, l'inopportunità di costruire gerarchie di valori su base storiografica da assumere a guida per l'operatività. In questa sequenza cronologica entrano anche le alterazioni e le patologie di degrado.

Ogni singola unità stratigrafica attesta inoltre un intervento che è genuino prodotto del contesto che lo ha prodotto, più o meno intenzionalmente. In quanto tale può o meno far riferimento ad un progetto o ad un sistema codificato di prassi costruttiva. Proprio questa non-necessità di una corrispondenza fra testimonianza fisica e codici del sapere e del saper fare induce a richiedere un ampliamento del lemma tecnica costruttiva verso quello di pratica costruttiva, intesa evidentemente in senso lato come documento di cultura. E' nella inestricabile concatenazione fra pratica e contesto (attinenza alla base del metodo della stratigrafia dell'architettura, dove per pratica s'intende un gesto consapevole che attiva risorse, mentre contesto è il luogo, sociale, economico, culturale ecc. cui tali risorse attingono)

\footnotetext{
${ }^{5}$ Fra le posizioni più avanzate che emergono dal dibattito disciplinare in corso in seno alle scuole italiane, merita ricordare quella che sostiene l'opportunità di praticare una conservazione intesa come gestione del mutamento e della trasformazione di un manufatto, in vista di nuove condizioni d'uso, di nuovi standard normativi e di sicurezza, privilegiando il rispetto rigoroso e la tutela del dato materiale così come di quello immateriale. Come corollario risulta anche necessario concepire diffusamente un'altra piccola rivoluzione; ossia accettare che il cantiere non rappresenti più un momento extratemporale in cui viene azzerato il tempo della vita del manufatto, bensì un momento in cui il progettista si cala in un'esistenza ininterrotta con le armi della manutenzione, della riparazione e dell'adeguamento.
} 
che si situa il tema che un po' impropriamente e sbrigativamente chiamiamo della "integrazione" e del suo carattere più o meno distintivo.

Le ragioni che sostengono questo intreccio sono più d'una. Ma almeno un pensiero va ribadito. In architettura il significato della stratificazione è radicalmente diverso rispetto a quello che normalmente distingue un bacino archeologico di scavo, dove ogni strato si sovrappone, indifferente, a quello che lo precede e che, di solito, è più antico. Qui, oltre al tratto materiale e alle opzioni tecnologiche proprie di ogni "strato", si racconta di una relazione (percettiva, funzionale, costruttiva, tecnologica, materiale ecc.) che questo strato stabilisce e attiva con quanto già esiste. L'essenza della questione è tutta in questa nonindifferenza. Non si tratta allora di reiterare vane dispute ideologiche sul fondamento dogmatico della conservazione. Semplicemente di considerare che in architettura la lettura di un quadro di relazioni rappresenta un dato imprescindibile per il suo apprezzamento. La rimozione anche di uno solo di questi strati produce una frattura cui non si può rimediare in alcun modo. Nemmeno con un sedicente restauro riparatore.

Conservare e -laddove indispensabile- provvedere a realizzare nuova architettura (con finalità di completamento stabilite dall'esigenza della buona conservazione e dalle concrete richieste del riuso), secondo un principio di distinguibilità regolato sul binomio pratica-contesto (ma, soprattutto, con intenzione di attivare una nuova forma di relazione con l'esistente), sembrerebbe allora un requisito perlomeno non contraddittorio con tali premesse.

Infine (ma altre considerazioni potrebbero ampliare non poco questo quadro di rapporti) le unità, nel momento in cui se ne prospetta l'aggregazione in interfacce di fase (anche come Unità associate), danno conto dei modi d'uso della fabbrica e delle loro modificazioni, individuando progetti e intenzioni, con i quali la progettualità che segue all'analisi può trovare un terreno in più di confronto, calibrando e possibilmente ottimizzando il grado di compatibilità.

A fronte di questa serie di considerazioni, che appaiono in sintonia con le elaborazioni più stimolanti della disciplina dell'intervento, come è noto si accompagnano da anni osservazioni critiche sulla difficoltà di creare un ponte forte e sicuro, consequenziale (ma -francamente- non vediamo la necessità di una consequenzialità), fra una tecnica (analisi stratigrafica) e gli obiettivi indagativi e/o interventivi di una branca dell'architettura. L'intervento di Bonelli del 1986 (BonelLI, 1986) è in gran parte condivisibile nei suoi aspetti tecnici, poi largamente discussi anche in seguito, mentre le questioni da lui sol- levate in merito alla liceità dell'utilizzo della tecnica stratigrafica in ambito architettonico-storiografico hanno dato impulso ad un fecondo dibattito e alla elaborazione di correttivi e di ampliamenti di senso. Interventi più recenti hanno sottolineato e rimarcato il carattere comunque limitato della conoscenza ottenibile dall'indagine diretta o indiretta e la necessità di procedure di lettura $\mathrm{e}$ di intervento che siano aggiornabili e che soprattutto non prevedano l'alterazione delle tracce e la distruzione dell'evidenza. Non bisogna poi dimenticare come l'enfasi connessa alla diffusione della tecnica di indagine archeologica anche in campo architettonico possa portare ad una sottovalutazione degli altri metodi di indagine. Come è stato più volte sottolineato, la logica strettissima e corroborante dell'analisi stratigrafica può esaltare lo strumento al di sopra degli obiettivi delle ricerche.

Infine non è superfluo ricordare come si debbano valutare e porre sotto particolare luce, individuando gli strumenti per studiarli, anche altri aspetti della stratificazione. In particolare quelli connessi alla sedimentazione dei dati immateriali e alla sequenza della mutazione dell'immagine dei manufatti e del contesto, che rimanda evidentemente a contributi non strettamente archeologici ed architettonici, che però non perché tali non possono essere esclusi aprioristicamente dalle valutazioni. In questo caso, il riferimento alla mentalità più che alla tecnica stratigrafica, aiuta a ricomprendere $i$ vari aspetti elencati in un orizzonte comune.

\section{NUOVI FRONTI}

Tanti nuovi ambiti di ricerca sono possibili e sono in procinto di essere tentati.

Si sente la necessità di incrementare la disponibilità della schedatura stratigrafica ad accogliere informazioni sempre maggiori e diversificate. Un processo che sarebbe senz'altro agevolato da una razionalizzazione della schedatura con sviluppo degli automatismi informativi.

Una formulazione di un sistema di collegamento schede-rilievi che porti ad una maggiore attenzione per le pratiche costruttive in termini non di tentativo di codificazione bensì di documentazione e confronto sarebbe utile a garantire un approfondimento degli aspetti storiografici (un fronte di ricerca che mira evidentemente ad un impiego più generalizzato della lettura archeologica per l'integrazione della lettura storica delle vicende della fabbrica).

La disciplina della storia dell'architettura sembra per ora sfuggita al rinnovellato coinvolgimento interdisciplinare suscitato dall'interesse per le tecniche di rilevamento 
archeologiche. La ragione di questa per ora mancata convergenza è in gran parte attribuibile alla tendenza a considerare la lettura stratigrafica come applicazione di un atteggiamento filologico esasperato, in grado però di raccogliere solo dati quantitativi, indifferenziati, e soprattutto parziali. Effettivamente il dato stratigrafico è spesso presentato (si pensi alle schede di unità stratigrafica e al diagramma matriciale di Harris) in una forma che per l'architetto e per lo storico appare al limite dell'utilizzabilità e anche, legittimamente, della comprensione; soprattutto nei casi, che rappresentano la norma, in cui le aree dove la lettura è possibile sono una piccola parte della fabbrica e dove la grande quantità di dati connessi all'uso quotidiano, alla manutenzione, alla cultura materiale (pur preziosissimi) soverchia di gran lunga le informazioni relative agli eventi costruttivi principali.

E' vero invece che è utile e possibile ricomprendere le informazioni che scaturiscono dalla lettura archeologica anche in un quadro semplificato, dove possono essere meglio evidenziate le fasi di costruzione e d'uso e dove al contempo sono possibili anche approfondimenti in merito agli aspetti post-deposizionali ${ }^{6}$.

La lettura archeologica sostiene inoltre il grande limite di non poter essere applicata che in frangenti molto particolari: edifici ruderizzati, o privi di intonaco, o sottoposti a drastici interventi. Ma tale limite è in molti casi solo apparente, giacché sembra possibile costruire e considerare una particolare casistica di giaciture di tipo archeologico che si possono ritrovare quasi in ogni edificio. Nelle fabbriche storiche, anche nelle più restaurate, continuano infatti quasi sempre a permanere alcune zone -generalmente nascoste o poco accessibili (o che divengono tali in seguito al progredire della stratificazione) - che si mantengono in qualche modo indenni dalle modifiche. In molti casi rappresentano addirittura delle zone "di bordo" dove cioè sono leggibili, quasi in sezione, le sovrapposizioni di materiali pertinenti a successivi interventi.

In alcuni casi è possibile parlare di vero e proprio deposito archeologico. Appartengono a questa categoria i riempimenti delle fosse di fondazione e dei rinfianchi delle volte, coevi alla costruzione ma arricchiti anche da depositi successivi.

Altri depositi sono gli strati di vespaio e di sottofondo in genere, nonché gli strati di coibentazione tradizionali. In quest'ultimo caso, diversamente da quanto avviene, per esempio, nella realizzazione di un intonaco o di un pavimento, il processo di stratificazione può prolungarsi

${ }^{6} \mathrm{Si}$ veda in proposito anche quanto espresso in Pertot, Tagliabue, Treccani, 1996; Pertot, 1997; Treccani, 1997. nel tempo con apporti regolari, casuali o intenzionali, di materiali di scarto od obsoleti.

Vi sono poi parti della fabbrica che - non essendo in vista - non sono soggette ad intonacatura o a regolarizzazione (neanche in caso di modifiche): si tratta, fra le altre, delle murature comprese nei sottotetti (zone di coronamento, frazioni sommitali di muri di spina), e delle parti di muratura comprese in intercapedini. Sono zone nascoste alla vista, dove più frequentemente si osservano le interfacce fra interventi successivi, dove si distinguono bordi che non è stato necessario regolarizzare od occultare, o dove l'abbassamento della quota di un solaio ha lasciato scoperti una vecchia cornice, tracce di intonaci, residui di decorazione pittorica, la linea dei vecchi travetti o una serie di elementi architettonici altrove rimossi o alterati.

Un particolare cenno meritano le canne fumarie, che sono elementi strettamente connessi all'organizzazione distributiva e al modello d'uso, e che hanno specifici rapporti stratigrafici con le murature.

Condizioni ottimali di visibilità stratigrafica si riscontrano infine, sebbene in misura minore, nei locali cantinati.

La considerazione della giacitura suggerisce metodi e modi della prospezione: scavo stratigrafico (laddove sia possibile e realmente opportuno) per quanto riguarda la prima categoria di giaciture considerata (fosse di fondazione, rinfianchi) e parte della seconda (vespai, riempimenti); rilievo stratigrafico, a vista, su fotografie, con supporti fotodigitali o con ausilio di metodi diretti e indiretti di rilevamento, per indagare le murature "nascoste".

Il rapporto fra tecniche di indagine archeologica e obiettivi dell'indagine storica sugli edifici stratificati, sovente gestito dall'architetto o dall'archeologo che operano la ricerca ai fini dell'intervento, segna non di rado una sorta di impasse che richiede un'analisi non semplice. A questo proposito bisogna ricordare che la descrizione e la presentazione delle note storiche riguardanti gli edifici considerati di maggior pregio viene tuttora affrontata (per tradizione ormai centenaria) prevalentemente su basi artistico/stilistiche, risentendo di una matrice profondamente legata ad alcuni aspetti dell'idealismo. Questa matrice in Italia è connessa con le modalità di nascita e di sviluppo della disciplina della storia dell'arte, da Adolfo Venturi in poi, con l'ordinamento del suo insegnamento nelle scuole e nelle università e, conseguentemente, con gli esiti delle ricerche. Un imprinting ancora così presente nel "bel paese" $\mathrm{da}$ rendere il settore tendenzialmente impermeabile a sollecitazioni di segno diverso che provengono da altri setto- 
ri. Le polemiche in merito sono tante e ben distribuite nei diversi settori disciplinari (Giovannoni e Venturi, Lugli e Lamboglia ...), ma ad oggi appaiono, più che superate, sopite.

Non di rado invece capita, che una campagna di rilevamento stratigrafico faccia emergere nuovi elementi storiografici, e che questi vengono ignorati o, nel caso di fasi secondarie, sentiti come ininfluenti ai fini del giudizio storico-artistico e utili al più a incrementare la sequenza cronologica, stante le scarse speranze di trovare riscontro nella documentazione archivistica.

La scelta di esperienze che si presenta brevemente in questa sede può dirsi -si spera- emblematica della potenzialità dello strumento stratigrafico, su fronti diversi: sia per una più capillare comprensione del manufatto, laddove l'assunzione di parametri stilistici troppo stretti, privi di confronto con il dato fisico, generi una lettura parziale o anche inesatta delle fasi costruttive, sia per un più corretto ed articolato inserimento e sviluppo dell'apporto progettuale.

\section{TRE INDICAZIONI DA ESPERIENZE DI LETTURA STRATIGRAFICA} Un primo caso riguarda la chiesa di Santo Stefano a Vimercate, alle porte di Milano ${ }^{7}$. Si tratta di un complesso articolato in due settori ben distinti costruiti in momenti diversi: un settore costituito dalle tre navate (e dalla facciata), che la critica da decenni considera (sulla base della presenza di grandi finestre a tutto sesto nella parte alta) come nucleo primitivo, al quale sarebbero stati aggiunti il presbiterio, con cripta, le tre absidi e le decorazioni esterne ad archetti pensili (proto)romanici e il campanile, ulti- mato, secondo documenti, nel 1261. Il rilievo stratigrafico ha evidenziato invece un chiaro rapporto di posteriorità della zona delle navate non solo rispetto a quella presbiteriale, ma anche rispetto al campanile. Quest'ultimo, ruotato rispetto all'asse delle navate, ha l'ingresso parzialmente occluso dalla testata del muro perimetrale della navata destra. Inoltre le grandi finestre della navata centrale (tamponate in seguito alla costruzione di una volta a botte nella navata centrale e oggi visibili solo nei sottotetti e dall'esterno) non sono disposte sui due lati in posizione simmetrica: quelle del lato destro tengono contro della presenza della mole del campanile (che occupa la prima campata della navata destra), che pertanto ben difficilmente può essere considerato aggiunta posteriore.

Nonostante queste indicazioni, recentissimi contributi a stampa hanno continuato a proporre le precedenti interpretazioni, di fatto trascurando le indicazioni stratigrafiche e geometriche rilevate e rinunciando a darne un'interpretazione. Non è un comportamento inconsueto, che si è riscontrato anche nel caso di monumenti ben più noti, studiati e restaurati ${ }^{8}$

Un altro caso che si richiama in questa sede (senza allegare immagini, ma solo per sottolineare un particolare aspetto metodologico) riguarda una campagna di studio avviata sulle murature del Castello Sforzesco di Milano, in realtà visconteo-sforzesco 9 . In seguito ai restauri condotti da Luca Beltrami a partire dal 1894 il complesso è stato spogliato delle parti aggiunte a partire dal XVI secolo ed

Scheda n.2 in De Grossi, 2000: 290-93

${ }^{8}$ Si veda il caso di Santa Maria del Tiglio a Gravedona (CO) nella rilettura di Stefano Della Torre (Della Torre, 1997).

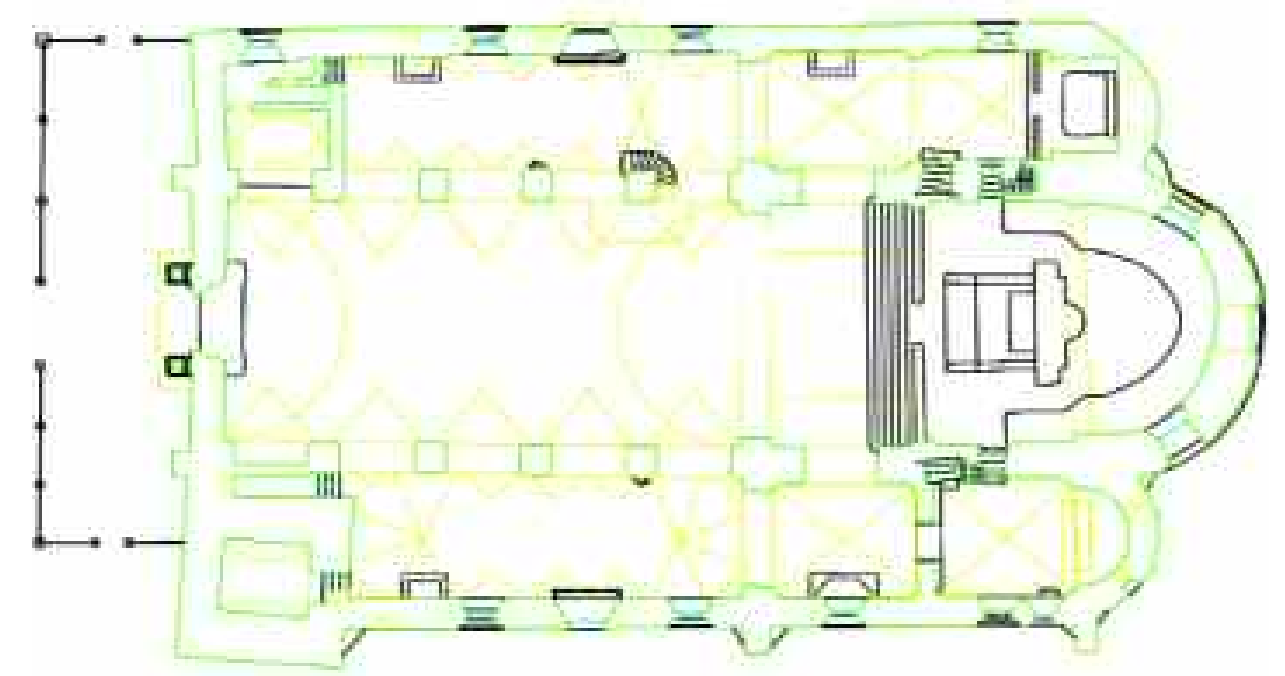

Fig. 1. Santo Stefano a Vimercate. Sezione orizzontale a quota $+1,50 \mathrm{~m}$. 
integrato con metodo per allora assai rigoroso fino ad assumere consistenza ed aspetto prossimi, almeno sul piano dell'immagine e dell'impianto, a quelli che avrebbe avuto prima della caduta della signoria degli Sforza. Per tracciare le linee del suo progetto Beltrami si avvalse al tempo di tutti gli strumenti disponibili: scandagli, rilievi, osservazioni stratigrafiche, ricognizione capillare delle fonti storiche e bibliografiche, straordinaria conoscenza della storia e della storia dell'architettura e dell'arte, unita a grande attitudine tecnico-professionale. A tutt'oggi gli studi di Beltrami rappresentano il punto di partenza per chiunque tenti ulteriori indagini sul complesso, ponendosi come principium auctoritatis che è stato assunto per più di un secolo come esaustivo nei confronti di ogni esigenza conoscitiva $^{10}$. In particolare, complice la mancanza di rilievi, non è stata indagata in nessun modo l'effettiva estensione delle strutture pre-sforzesche, né sono stati chiariti alcuni dubbi sui limiti e sui collegamenti fra le diverse parti del castello.

Una ricerca intrapresa recentemente ha preso in esame un particolare indicatore, costituito dalla presenza di buche pontaie diagonali, verificandone la presenza, in allineamenti non casuali, in diverse parti delle cortine. Ipotizzando che, come quasi sempre avviene, la presenza di fori pontai inclinati sul piano orizzontale della muratura corrispondesse all'esigenza dei mastri muratori di rendere possibile la prosecuzione dei ponti lignei in prossimità di un angolo del fabbricato, ne deriva un'originale mappatura delle cortine del Castello che mostra un'articolazione ancora tutta da spiegare, ma sicuramente da rapportarsi a preesistenze trecentesche o comunque anteriori al $1450^{11}$.

Un ultimo riferimento riguarda esperienze didattiche condotte in questi ultimi anni con l'intento di integrare le fasi conoscitive basate sull'impiego della metodologia di

\footnotetext{
9 Nell'ambito delle esercitazioni degli studenti del Laboratorio di Restauro Architettonico presso la I Facoltà di Architettura del Politecnico di Milano Leonardo, sono stati elaborati rilievi stratigrafici (con campagne di rilievo mensiologico dei laterizi) e progetti di conservazione e di riuso sui seguenti siti: torrioni circolari, cortine su piazza d'armi, strada coperta (tratte sud-ovest e nordovest), difese esterne, Porta del Soccorso (a.a. 1999-2000); battiponte e rivellino del Carmine, battiponte verso il parco, fossato morto, locali ex Scuole d'Arte ed ex Direzione Biblioteche rionali, strada coperta (tratta nord-est), torre Umberto I (a.a. 2000/01).

${ }^{10}$ Il testo di Beltrami (Beltrami, 1894) è tuttora vasto e prezioso repertorio di informazioni documentarie e di riflessioni critiche svolte dall'autore.

${ }^{11}$ Un primo rapporto (G. Pertot, La fabbrica medioevale: sopravvivenze ed integrazioni) verrà pubblicato a breve in un testo dedicato a diversi aspetti della storia del Castello di Milano, curato da Maria Teresa Fiorio.
}

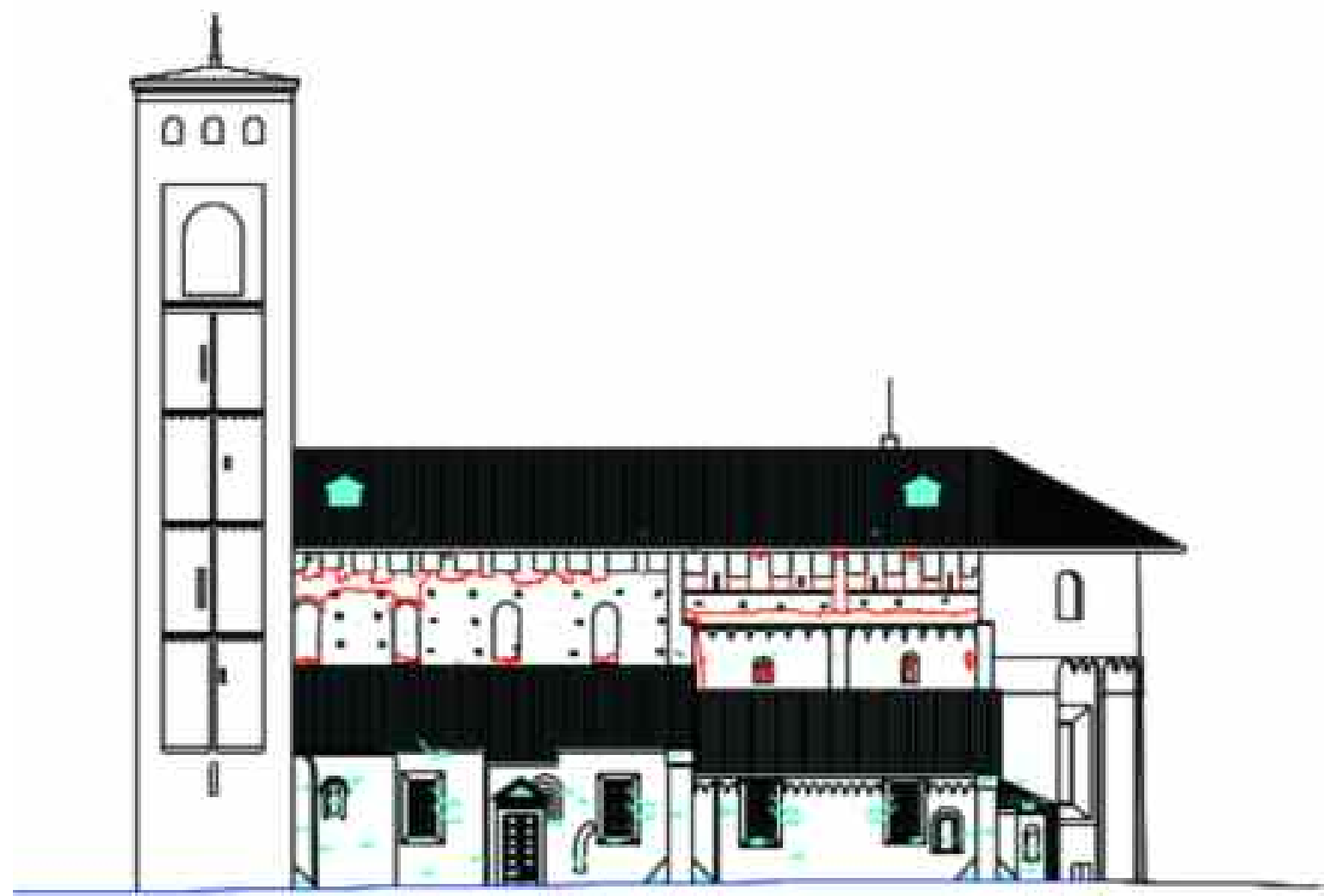

Fig. 2. Santo Stefano a Vimercate. Prospetto meridionale. Analisi stratigrafica 


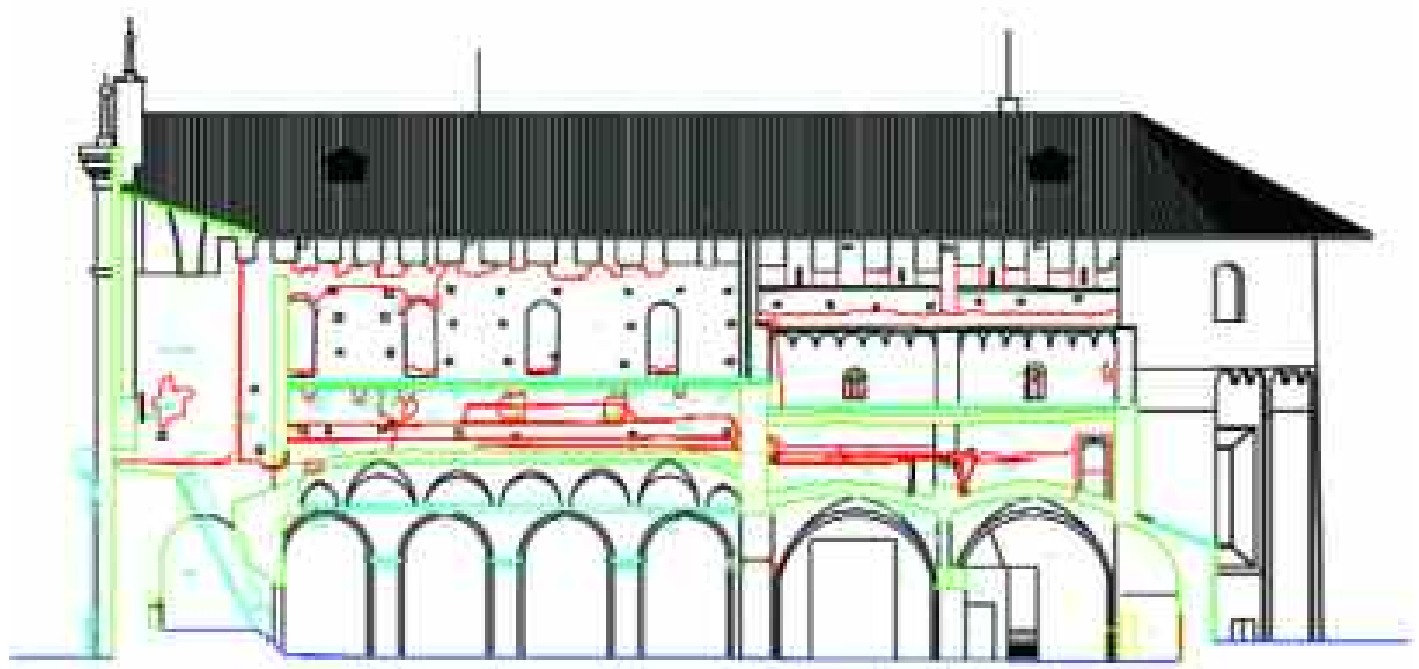

Fig. 3. Santo Stefano a Vimercate. Sezione verticale sulla navata meridionale. Analisi stratigrafica

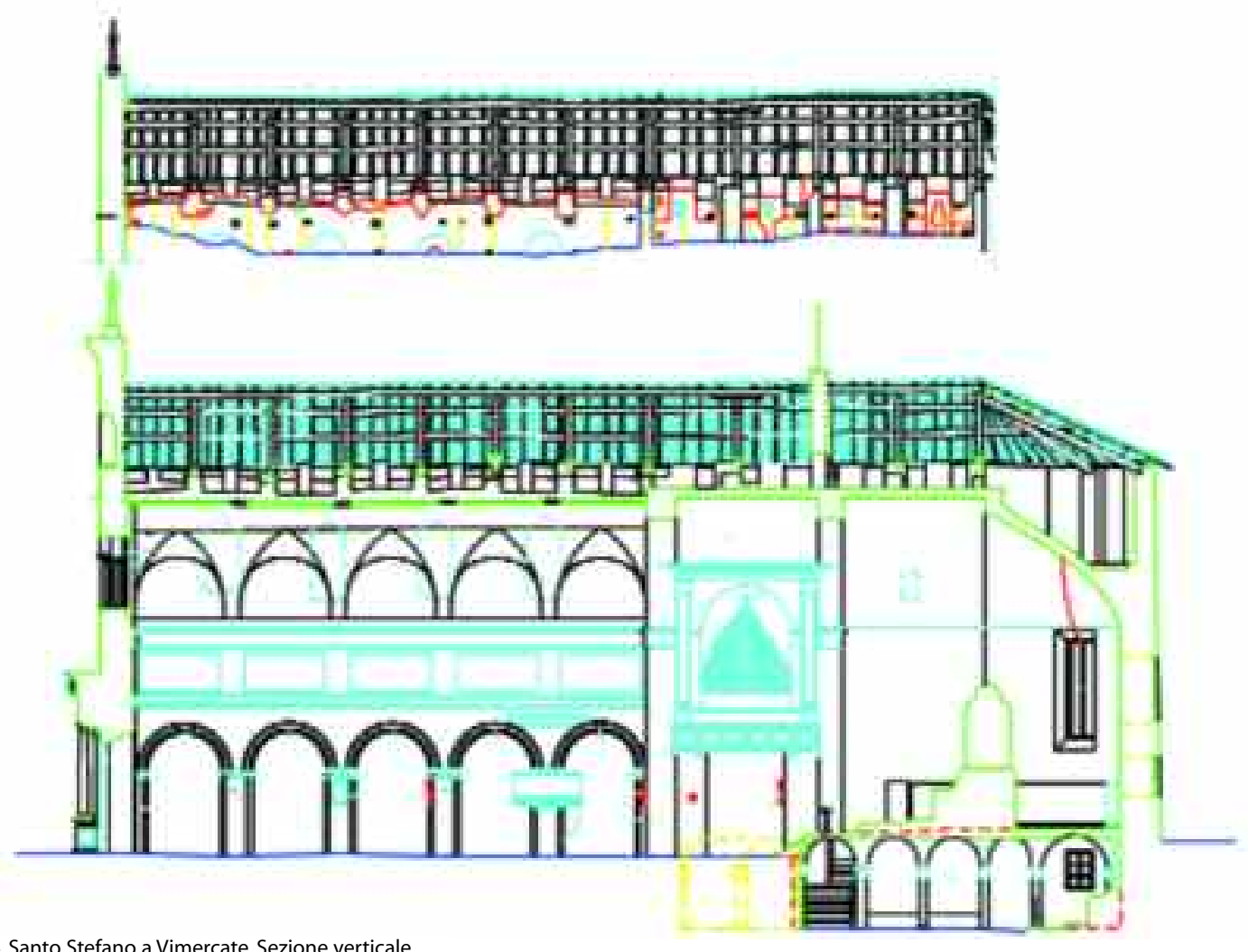

Fig. 4. Santo Stefano a Vimercate. Sezione verticale longitudinale e sezione a ridosso della navata

settentrionale (particolare della zona del sottotetto). Analisi stratigrafica 
rilevamento stratigrafico e le fasi progettuali. Nel caso che si presenta in dettaglio (con la riproduzione di diverse tavole di analisi e di progetto) l'apporto di maggior interesse deriva da un tentativo di articolare la proposta per il riuso di un edificio estremamente stratificato con la lettura delle interfacce di fase (De Grossi, Panarese, 1996; De Grossi, 1997). L'edificio in questione è la chiesa di Castelletto Monastero a Castelletto Cervo, in provincia di Biella, nella regione Piemonte. La chiesa venne eretta sui resti di un insediamento romano, su un sito rilevato, strategicamente importante per il controllo del territorio. Il complesso nacque come comunità benedettina per poi divenire priorato cluniacense tra il 1087 ed il 1095. Nel 1127 una donazione permise una profonda ristrutturazione, che comportò probabilmente l'aggiunta di un nartece, poi sopraelevato fino ad eguagliare l'altezza della facciata. La chiesa subì poi nuovi interventi, fino alla seconda metà del XVII secolo, quando venne sottoposta ad una profonda ridefinizione: venne rifatta tutta la parte absidale, le volte delle navate laterali furono ricostruite ad una quota inferiore e a crociera, mentre la navata centrale venne coperta da volta a botte. Negli spazi ricavati nel sottotetto (abbassando le volte delle navatelle) e in quelli del nartece vennero ricavati gli uffici e l'abitazione parrocchiali. Verso il 1950 sono stati invece effettuati interventi per il consolidamento della struttura, mediante l'inserimento di elementi (travi, corree...) in cemento armato.

La suddivisione dell'evoluzione architettonica e funzionale della chiesa in fasi appartenenti ad epoche specifiche e caratterizzate da tecniche specifiche è stata effettuata sulla base del rilevamento stratigrafico, incrociando i dati con le informazioni derivate dalla ricognizione archivistica. Su questa base sono state individuate le patologie di degrado, anch'esse poste in relazione agli eventi costruttivi/distruttivi (si è posta in relazione, per esempio, la dinamica del degrado statico con le diverse opere di modifica della configurazione originaria).

Posto che l'analisi stratigrafica ha permesso di cogliere la complessità e l'unicità del complesso, qualificandolo come un unicum continuo, sommatoria di molteplici apporti, il progetto di riuso ha proposto un percorso articolato all'interno dei vari spazi, inserendo nuove destinazioni d'uso e nuove strutture, alle quali è stato affidato anche il compito di risolvere alcuni dei problemi statici della grande fabbrica.

In particolare è stato studiato un nuovo sistema di accesso alla chiesa e agli spazi superiori inserendo nuovi elementi nel nartece, fra i quali una scala, un ascensore ed una passerella di collegamento. Scala e ascensore sfruttano l'attuale mancanza di solai intermedi nel nartece stesso, mentre la passerella si assesta alla quota del vecchio livello d'uso. Elementi guida del progetto sono stati la volontà di evitare il contatto delle nuove strutture con le murature, scegliendo invece per gli ancoraggi le unità stratigrafiche pertinenti all'ultima fase costruttiva, ossia le parti in cemento armato, lascito di un intervento discutibile e irreversibile, ma comunque in grado, per le sue caratteristiche, di fornire sufficiente resistenza ai nuovi carichi e di ripartirli in modo ottimale sulle murature in laterizi.

Il linguaggio scelto per i nuovi elementi è dichiaratamente contemporaneo: la struttura principale è in acciaio. La passerella è costituita da una doppia trave in lamiera pressopiegata, sospesa con tiranti ad una trave a sezione variata in legno lamellare, ancorata ai cordoli in cemento armato esistenti. Sotto la passerella, collegata agli stessi tiranti, un'altra trave riveste la funzione di ancoraggio di sicurezza per le sottostanti volte del nartece, oggi in precario equilibrio.

\section{Bibliografía}

BELLINI A., 1996, A proposito di alcuni equivoci sulla conservazione, TeMA, 1, pp.2-3.

Beltrami L., 1894, Il Castello di Milano (Castrum Portae Jovis) sotto il dominio dei Visconti e degli Sforza, MCCCLXVIII-MDXXXV, Milano.

BONELLI R., 1986, Archeologia stratigrafica e Storia dell'architettura, Architettura, storia e documenti, 2, pp. 5-10.

De Grossi F., PANARESE S., 1996, Progetto di conservazione e riuso museale della chiesa dei Santi Pietro e Paolo a Castelletto Cervo (Biella). Proposta di metodo, tesi di laurea, Facoltà di Architettura del Politecnico di Milano, relatore prof. G.P.TRECCANI, a.a. 1995-96.

De Grossi F., 1997, Stratigrafia e progetto di riuso museale: la chiesa di Castelletto Monastero a Castelletto Cervo (Biella), АNAГKH, 17-18, pp. 202-9.

De Grossi F., 2000, Archeologia e progetto. Alcune esperienze didattiche, in Treccani G. P. (a cura di), Archeologie, restauro, conservazione, Milano, pp. 247-309.

Della TORRE S., 1997, S. Maria del Tiglio: la 'archeologia sperimentale' nella tradizione del restauro architettonico, Archeologia dell'architettura, II, pp. 199-205

OTERI A.M., 2000, L'archeologia dell'architetto. Restauri d'archeologia nelle riviste d'architettura, in TrecCANi G. P. (a cura di), Archeologie, restauro, conservazione, Milano, pp. 177-94.

PERTOT G., 1997, Dall'analisi stratigrafica al progetto di conservazione e riuso. Esperienze didattiche e nuove procedure analitiche, in Patrimonio archeologico e progetto architettonico e urbano (atti del convegno, Milano, 21-22 maggio 1996), Firenze, pp. 59-62.

Pertot G., 2000, Temi del restauro, Notizie degli scavi di antichità (18761997), in TrecCani G.P. (a cura di), Archeologie, restauro, conservazione, Milano, pp. 97-175.

Pertot G., Tagliabue R., Treccani G.P., 1996, Sperimentazioni didattiche tra archeologia stratigrafica e conservazione del costruito, in Dal sito archeologico all'archeologia del costruito. Conoscenza, progetto e conservazione, Atti del convegno di studi, Bressanone, 3-6 luglio 1996, Padova, pp. 61-74.

TrECCANI G.P., 1997, Sull'utilità (e il danno) della stratigrafia archeologica nel progetto di conservazione e di riuso, АNАГКH, 17-18, pp. 197-201.

TreCCANi G.P., (a cura di) 2000, Archeologie, restauro, conservazione, Milano. 


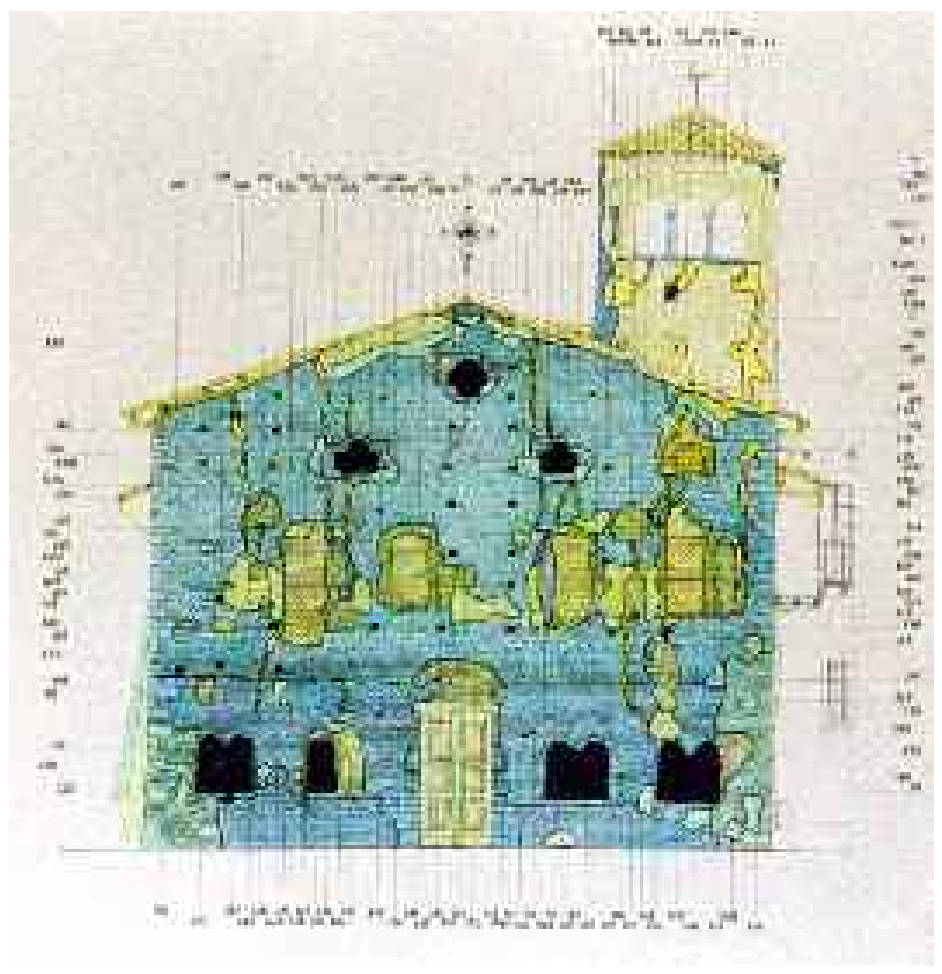

Fig. 5. Castelletto Monastero. Facciata. Analisi stratigrafica e Interfacce di fase

Fig. 6. Castelletto Monastero. Fianco meridionale.

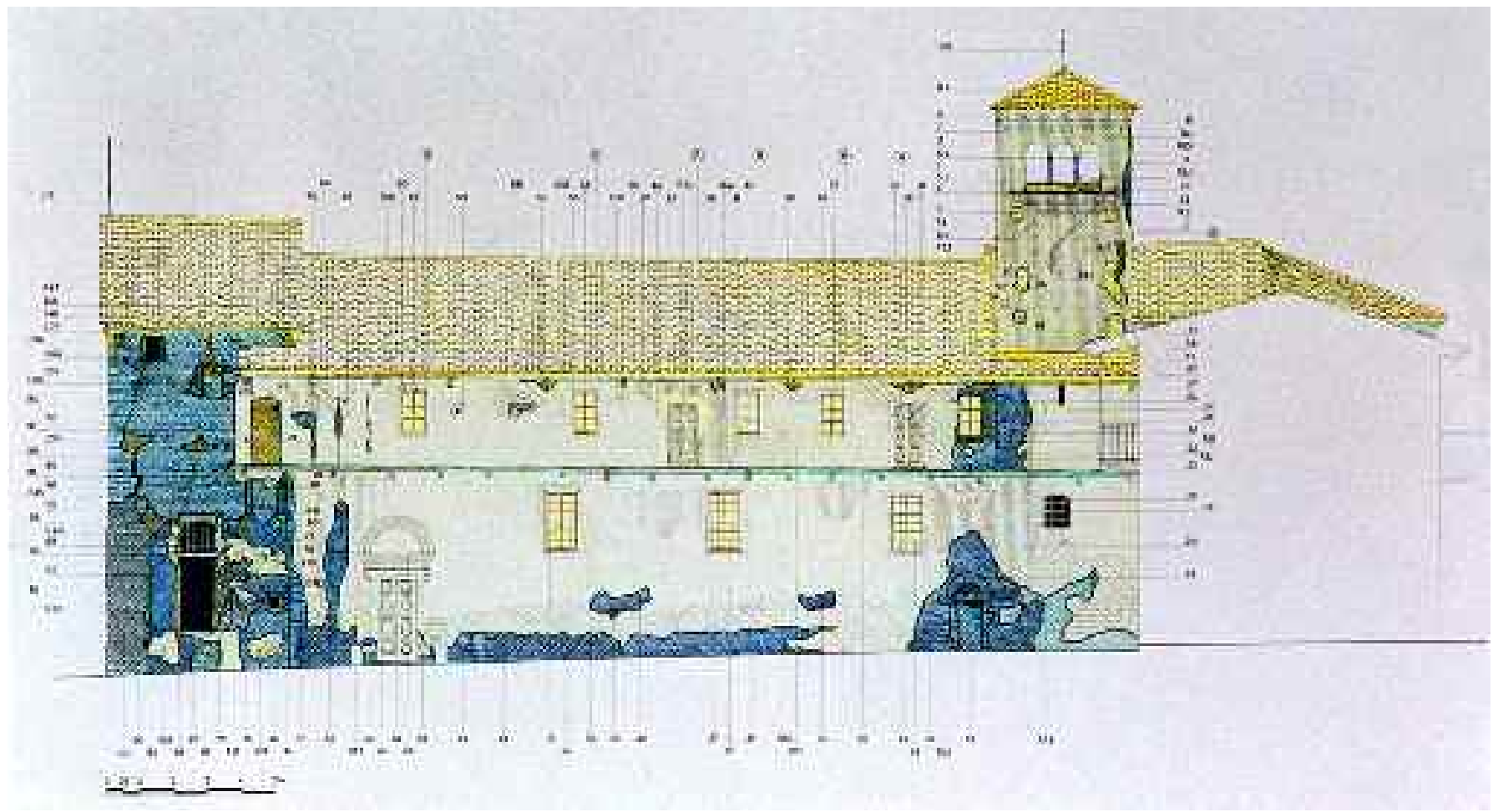



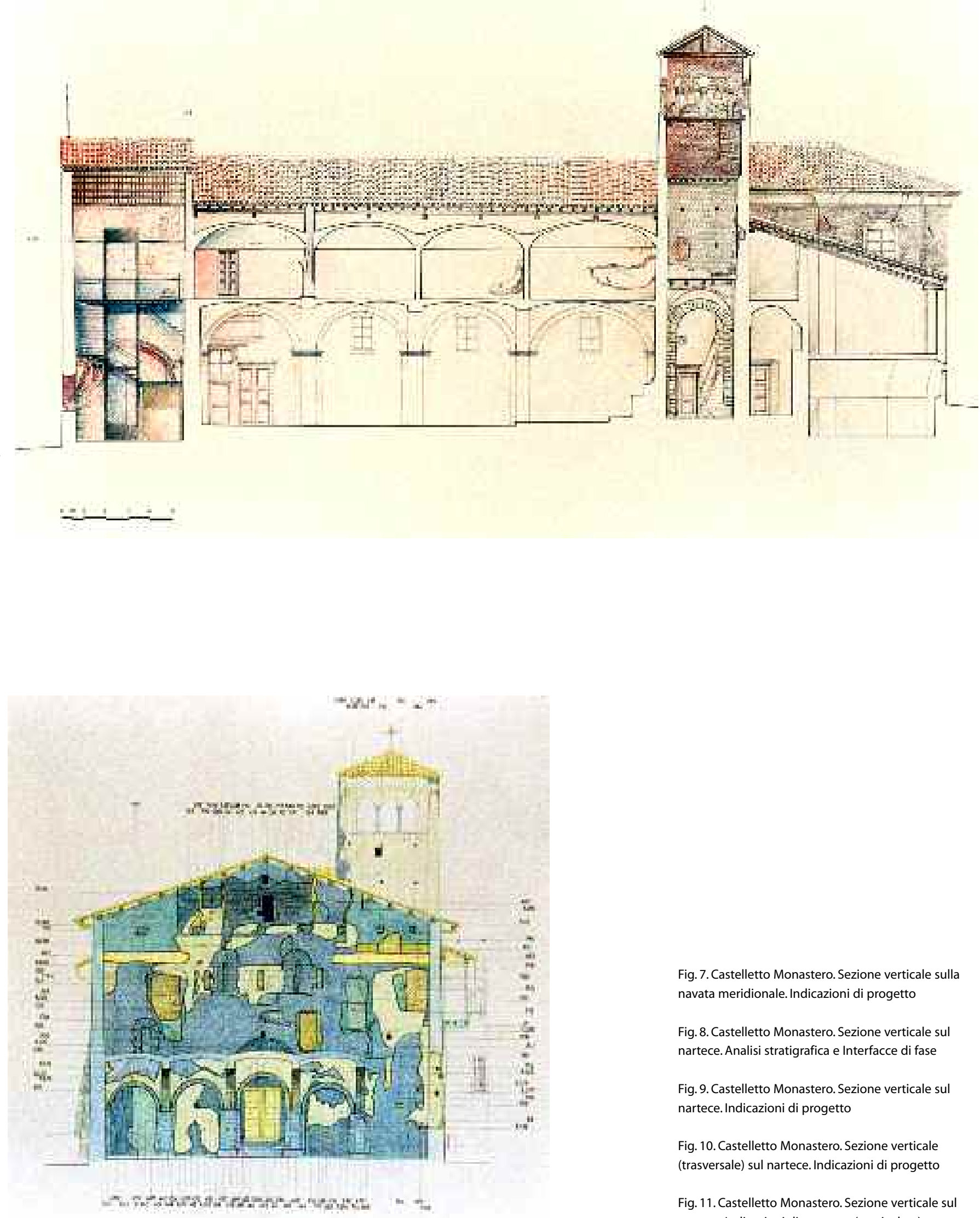
Fig. 7. Castelletto Monastero. Sezione verticale sulla
navata meridionale. Indicazioni di progetto

Fig. 8. Castelletto Monastero. Sezione verticale sul nartece. Analisi stratigrafica e Interfacce di fase

Fig. 9. Castelletto Monastero. Sezione verticale sul nartece. Indicazioni di progetto

Fig. 10. Castelletto Monastero. Sezione verticale (trasversale) sul nartece. Indicazioni di progetto

Fig. 11. Castelletto Monastero. Sezione verticale sul nartece. Indicazioni di progetto (particolare) 

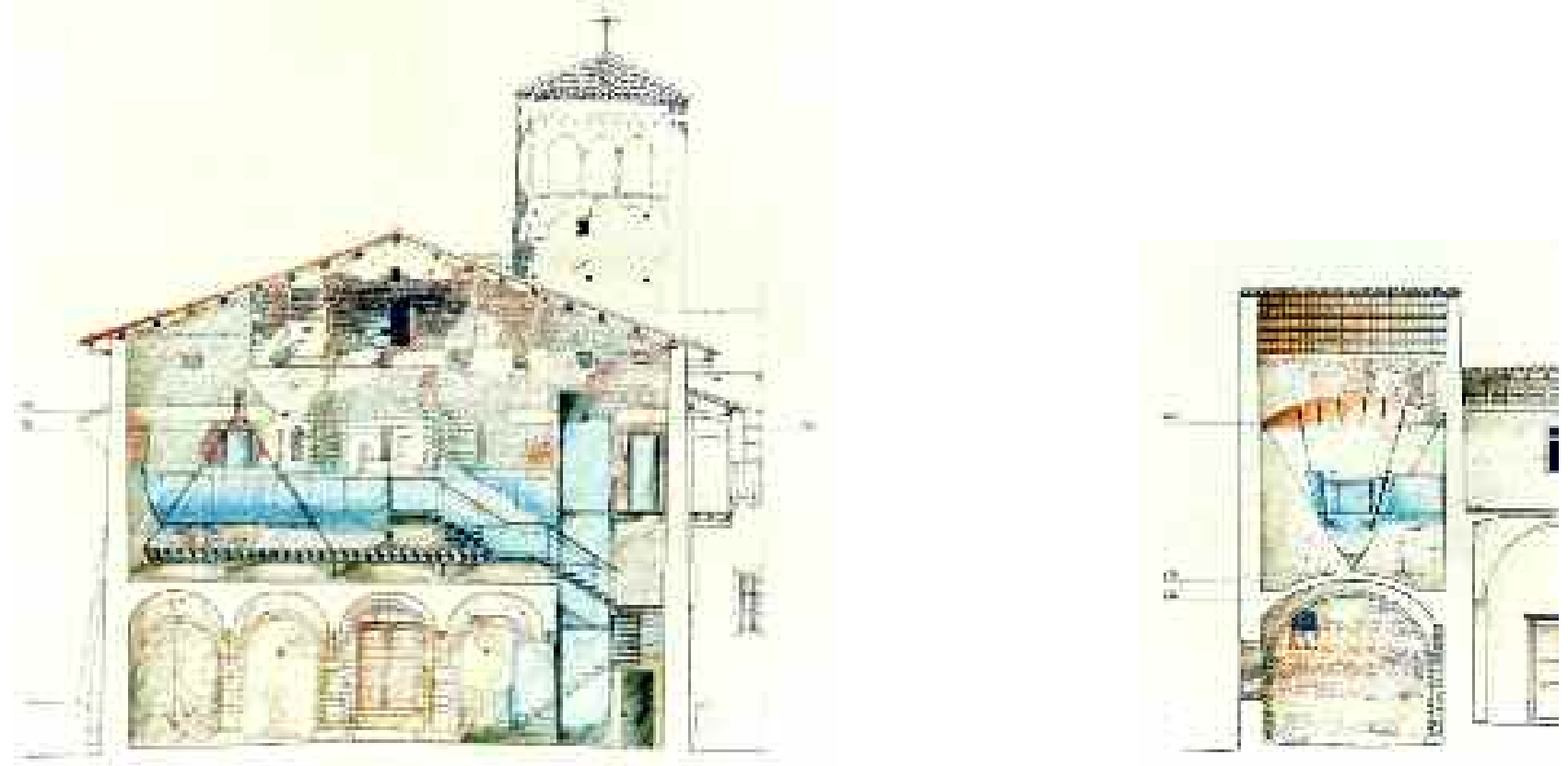

10
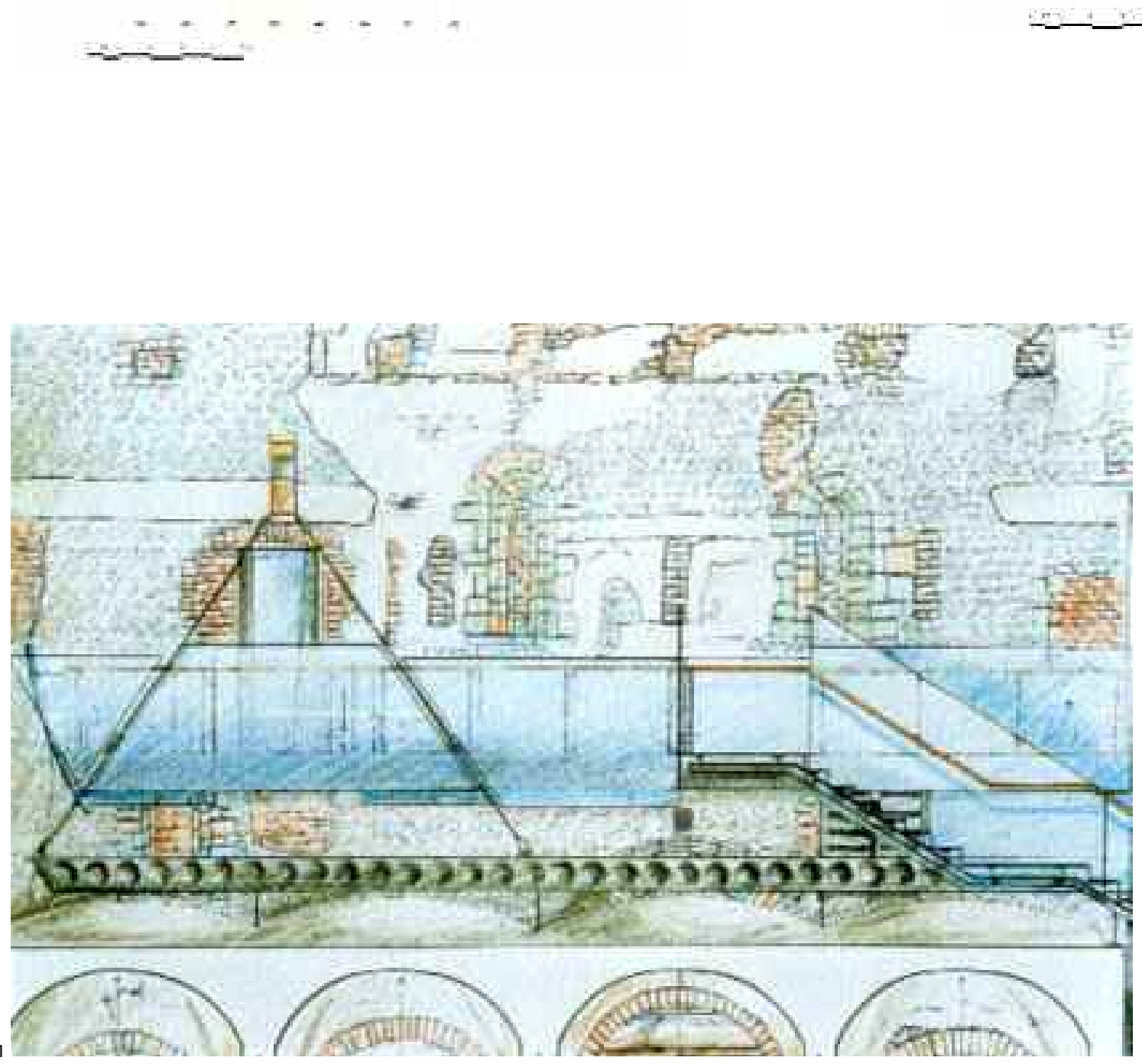